\title{
3-D PRINTED PALM ADAPTORS FOR HAND-ARM VIBRATION MEASURES: TRANSMISSIBILITY PROPERTIES AND MISALIGNMENT REDUCTION WHEN USED WITH GLOVES
}

\author{
ANDREA ANTONUCCI ${ }^{1}$, GIANLUCA COLTRINARI ${ }^{1}$, PIETRO NATALETTI ${ }^{2}$, \\ RAOUL DI GIOVANNI ${ }^{2} \&$ DARIO LIPPIELLO ${ }^{1}$ \\ ${ }^{1}$ University of Rome 3, Italy \\ ${ }^{2}$ Italian Workers Compensation Authority (INAIL), Italy
}

\begin{abstract}
The estimation of the transmissibility of antivibration gloves requires the use of handheld adaptors for holding the accelerometer and assure that it is correctly positioned at the palm level of the hand. The marketplace proposes only few adaptors, specially made for hand-arm vibration measures, so researchers often had to make their proper homemade adaptors to performing their experiments on the gloves' transmissibility. A way to easily build hard-plastic adaptors is given by the possibility to use 3D printers, but in the literature, there is a lack of information about their properties. This paper investigates the properties of 3-D printed adaptors, confronting different materials among the most used (PLA, ABS, and Nylon), and evaluates possible changes of their performance at different infill densities. Results show that 3-D printed adaptors can be a valid alternative to those present on the market. At middle-low frequencies $(10 \mathrm{~Hz}-400 \mathrm{~Hz})$ their transmissibility values are inside the range \pm 0.05 . No significant differences were found between the transmissibility of those printed at $50 \%$ or $100 \%$ of infill, especially when the acceleration is $\mathrm{W}_{\mathrm{h}}$ weighted. It has been also experimented a simple solution that aims to reduce possible misalignments of the adaptor when inserted inside the glove. Two external landmarks were designed in order to have a visual check of the position of the adaptor. The first, coming out from the glove through a little slit, was made for checking about the rotation on the $\mathrm{Y}-\mathrm{Z}$ plane, while the second, parallel to the wrist, was made for checking rotation on the $\mathrm{X}-\mathrm{Z}$ plane. The statistical analysis, among 30 measures performed by three operators holding the instrumented handle of a mono-axial shaker, supports the idea that the first landmark can help to achieve a better alignment of the adaptor, limiting its rotation along the $\mathrm{Y}-\mathrm{Z}$ plane. Lesser evidence resulted on the reduction of the misalignment on the $\mathrm{X}-\mathrm{Z}$ plane, due to the use of the second landmark. A possible reason is the small thickness of the ordinary leather glove used in the tests, which produces too little misalignments angles for being checked by the adaptor's landmark. Some further tests can be carried out using anti-vibration gloves, generally made of thicker and softer materials.
\end{abstract}

Keywords: antivibration gloves, hand-arm vibration, vibration transmissibility, handheld adaptors, $3 D$ printers, $P L A$.

\section{INTRODUCTION}

Prolonged exposure to hand-arm vibration can be at the origin of several illnesses, generating a set of symptoms generally called hand-arm-vibration syndromes (HAVS). Many tools usually used in workplaces can generate high levels of vibration such as chainsaws, pneumatic rock drills, pneumatic breakers, impact wrenches, orbital sanders, etc. [1]-[3], especially when workers need to use these tools for several hours a day. It thus results difficult to limit the exposure to vibration, calculated as recommended by the ISO Standard 5349-1 [4], below the limits required in many countries [5], [6].

The use of personal protection equipment, such as antivibration gloves (AV), can help to protect workers. The ISO Standard 10819 [7] specifies the properties that gloves must-have in terms of transmissibility, evaluated as the ratio between the vibration measured at the palm level inside the glove and that measured in the same conditions in a bare-adaptor test. To be 
labeled as an AV glove, the transmissibility must be lesser than or equal to 0.9 in the middlefrequency range $(25-200 \mathrm{~Hz})$ and lesser than or equal to 0.6 in the high-frequency range $(200-1,250 \mathrm{~Hz})$. Anyway, the efficacy of AV gloves is controversial, since it depends on many factors, often difficult to take all into account, both when the tests are performed as specified by the ISO 10819:2013 Standard, then much more, when the measurements are taken using real tools [8], [9].

Among all the different factors that can influence the evaluation of the transmissibility of gloves, two of them can introduce systematic relevant errors that can invalidate the results of the measures. These are the geometrical and mechanical characteristics of the adaptor used to locate the accelerometer at the palm level [10], [11], and the possible misalignments that the adaptor can have with respect to the direction of the vibration [12], [13].

The ISO 10819:2013 specifies the characteristics (shape, dimensions, weight, materials, and transmissibility) that handheld adaptors must have for being used in the measures of gloves' transmissibility. Among the allowed materials it is admitted also the use of hard plastic.

The marketplace proposes few handheld adaptors, specially made for hand-arm vibration measures, as specified by the ISO 5349:2001. For this reason, these adaptors often have dimensions dissimilar from those specified by the ISO 10819:2003 standard. Moreover, in most cases, they are designed to hold only a specific accelerometer. The errors introduced by these adaptors, even when used for hand-arm vibration measurements, are often greater than those made for gloves' transmissibility tests [10], [14], [15]. It follows that researchers often had to resort to making homemade adaptors to perform their experiments on gloves' transmissibility, often using metal materials [12], [14].

A way to easily build hard-plastic adaptors is the use of 3-D printers. Even though this solution has been already experimented in some tests on hand-arm vibration [11], [16], in the literature there is a lack of information regarding the properties that 3-D printed adaptors have in terms of transmissibility, both when different filaments are used and when changes the infill percentage, that is one of the main parameters required to be set when 3-D printers are used.

In the light of the previous considerations, this paper aims to investigate more deeply the properties of 3-D printed adaptors, confronting different materials among the most used, and evaluating possible changes of their performance at different infill densities.

Regarding the adaptors' misalignments and their consequences on the transmissibility's evaluation, several studies were made along the years, both estimating the magnitude of the errors [1], [13], and trying to find out solutions for their limitation, such as the use of triaxial accelerometers [17]-[20]. In this paper, we also experimented a simple solution that aims to reduce possible misalignment of the adaptor when inserted inside the glove for antivibration transmissibility tests.

\section{MATERIALS AND METHODS}

Starting from the dimensions provided by the ISO 10819:2013 [7], an adaptor with little modifications (1.45 mm higher) was designed in order to be able to contain a $10 \mathrm{~mm}$ cube accelerometer. Fig. 1 reports both the specified dimensions of the ISO Standard (in black) and the modifications made (in red). All the quotes indicated as "ISO" are the same prescribed by the 10819:2013 Standard. A 3-D model was then drawn and printed with an FDM (Fused Deposition Modeling) printer, using a $1.75 \mathrm{~mm}$ filament and $0.4 \mathrm{~mm}$ nozzle. Three different materials (PLA, ABS, and Nylon), each one printed with 2 different infill densities $(50 \%$ and $100 \%)$, were used for making the adaptors. These materials, commonly used with 3-D printers, have different mechanical and chemical properties [21]. PLA 
(Polylactic Acid) is a vegetable-based fully biodegradable plastic material, probably the most commonly used for FDM printers. It has higher stiffness compared to the others (Young's Modulus $(\mathrm{E})=800 \mathrm{MPa})$, but less strength (Ultimate Tensile Strength $(\sigma \mathrm{UTS})=25 \mathrm{MPa})$. The ABS (Acrylonitrile Butadiene Styrene), is a thermoplastic polymer, more flexible than the PLA $(\mathrm{E}=1.300 \mathrm{MPa})$, but it generally provides higher strength $(\sigma \mathrm{UTS}=45 \mathrm{MPa})$; while nylon provides the least stiffness $(E=1.500 \mathrm{MPa})$ but the highest strength $(\sigma \mathrm{UTS}=50 \mathrm{MPa})$. The weights of the printed adaptors at $100 \%$ of infill including the accelerometer, are as follow: PLA $14.3 \mathrm{~g}$; ABS $12.4 \mathrm{~g}$; Nylon $13.3 \mathrm{~g}$.

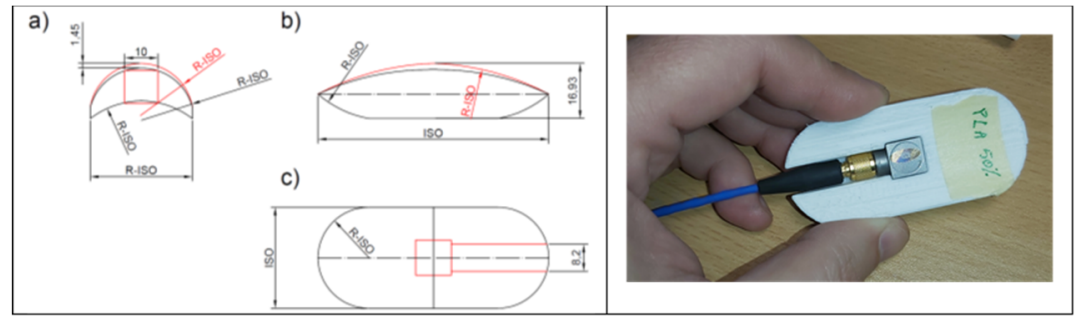

Figure 1: Dimensions of the printed adaptor.

We tested also other two adaptors. The first one was made of polypropylene ( $\sigma \mathrm{UTS}=$ $75 \mathrm{MPa} ; \mathrm{E}=1470 \mathrm{MPa} ; 10.5 \mathrm{~g}$ ) fabricated using the injection molding technique, with sizes conforming to the prescribed dimensions of the ISO 10819:2013 Standard. The second was a commercial T-shape fingers-held metal adaptor, made of anodized aluminum, with dimensions of $90 \times 22.5 \mathrm{~mm}$, and a weight of $24 \mathrm{~g}$. This adaptor is one of those generally provided on the market for hand-arm vibration measurements according to the Standard ISO 5349-2 [22].

A single-axis shaker (RMS-SW-1508/1) was used for the tests of the transmissibility. It was set to generate a broadband random vibration spectrum from 10 to $400 \mathrm{~Hz}$, according to the acceleration spectrum shown in Fig. 2. Although the fundamental natural frequency of the instrumented handle of the shaker was indicated to be above the frequencies range prescribed by the ISO 5349-1 [4], during the set-up a resonance phenomenon was found on the instrumented handle around $800 \mathrm{~Hz}$. For this reason, we limited the spectrum of the tests to $400 \mathrm{~Hz}$, considering the results valid only for low-middle frequency vibrations.

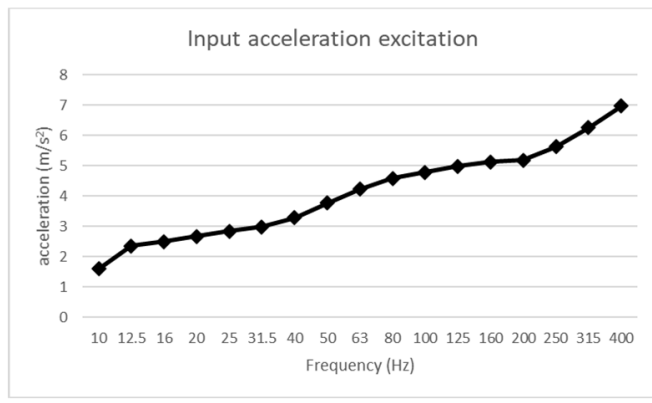

Figure 2: Broadband random vibration spectrum of the shaker. 
Although the frequency range used in this experiment does not cover the one required by the ISO Standard, it results quite sufficient for the purposes of this study, since the vast majority of powered hand tools fall within this frequency range [23]. Moreover, the frequency weighting at the frequencies above $400 \mathrm{~Hz}$ is less than $4 \%$ of the peak weighting value.

Each adaptor was fixed to the instrumented handle using a hose metal clamp, putting a little rubber layer between both the contact surfaces, as shown in Fig. 3. The tightening force was fixed at $80 \mathrm{~N}$ through the use of the screw of the clamp. The value of the force was checked by two force sensors located inside the instrumented handle.

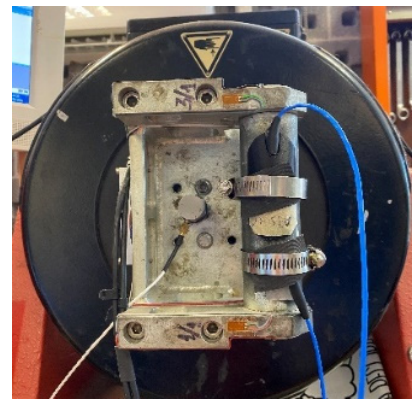

Figure 3: Instrumented handle.

For the measures of the vibration of all the printed adaptors and the metal one, a three axial $10 \mathrm{~mm}$ cubic accelerometer (SEN $040 \mathrm{~F}$ ) was used, weighing $5 \mathrm{~g}$; while for the test on the adaptor in polypropylene, it was used a three-axial accelerometer (SEN 026) $7 \times 12 \times$ $12 \mathrm{~mm}$, weighing $3.1 \mathrm{~g}$. In order to have a better estimation of the acceleration produced by the shaker, other than that located on the plate of the shaker, another 3 axes accelerometer (SEN $40 \mathrm{~F} ; 10.2 \times 19.6 \times 10.2 \mathrm{~mm} ; 5 \mathrm{~g})$ was fixed directly on the external surface of the instrumented handle, close to the adaptor.

A total of five tests were performed for each of the adaptors, each one lasting 30 seconds. Although we used three-axial accelerometers, after having verified that the components of the acceleration along the $\mathrm{X}$ and $\mathrm{Y}$ axes were not significant, we considered only the $\mathrm{Z}$-axis acceleration component for the estimation of the transmissibility of the adaptors. For each test, the baseline transmissibility at each frequency was computed from:

$$
T_{R(f) \_A}=\frac{a_{z(f) \_ \text {Bare_Adaptor }}}{a_{z(f) \_ \text {Handle }}},
$$

where $T_{R(f)-A}$ is the transmissibility of the adaptor at the single frequency $(f)$, and for the same frequency, $a_{z(f) \text {-Bare-Adaptor }}$ is the acceleration along the $\mathrm{Z}$ axis measured by the accelerometer positioned inside the adaptor, and $a_{z(f) \text {-Handle }}$ is the acceleration along the $\mathrm{Z}$ axis of the accelerometer positioned on the instrumented handle.

The total average transmissibility among all the frequencies was then calculated using the following equations, in both of the cases of linear and $\mathrm{W}_{\mathrm{h}}$ weighted acceleration:

$$
\bar{T}_{R L_{-} A}=\frac{\sqrt{\sum_{f} \bar{a}_{z(f) \_ \text {Bare_Adaptor }}^{2}}}{\sqrt{\sum_{f} \bar{a}_{z(f) \_ \text {Handle }}^{2}}},
$$




$$
\bar{T}_{R W h \_A}=\frac{\sqrt{\sum_{f} \bar{a}_{z(f) \_ \text {Bare_Adaptor }}^{2} \cdot W_{h(f)}^{2}}}{\sqrt{\sum_{f} \bar{a}_{z(f) \_ \text {Handle }}^{2} \cdot W_{h(f)}^{2}}},
$$

where in this case $\bar{a}_{z(f)}^{2}$ is the average acceleration of all the five tests for each material in the same conditions (bare adaptor and handle), and $W_{h(f)}$ is the frequency weighting factor defined in the ISO 5349-1 (2001) [4].

Another series of tests were also made to reduce the possible misalignment of the adaptors when used inside gloves. To facilitate the correct position of the accelerometer, when used with gloves, the ISO 10819:2013 suggests to place a slit in the seam of the glove between the thumb and index finger for visually check the position of the adaptor inside the glove. Starting from what suggested, and considering what already done in another experiment that needed external references to achieve a good position of the adaptor [11], we drew a protrusion along the longitudinal axis of the PLA 70\% infill adaptor, for making easier to check for possible misalignments respect to the $\mathrm{X}-\mathrm{Z}$ plane (Fig. 4).

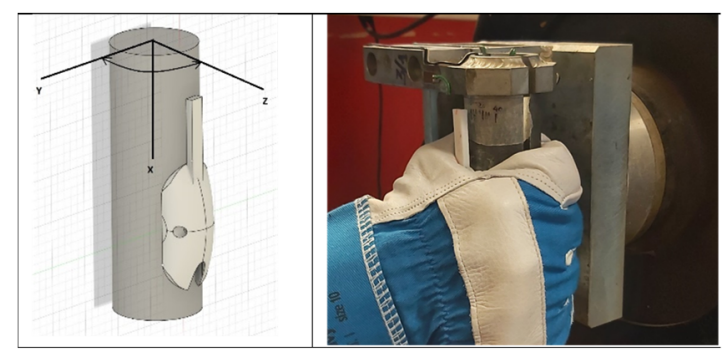

Figure 4: Adaptor's longitudinal landmark

Some other tests were also made using a second protrusion, draw along the transversal axis of the adaptor. In this case, the external referment was made for checking about possible misalignments with respect to the $\mathrm{Y}-\mathrm{Z}$ plane Fig. 5. These misalignments could mainly happen due to the compression of the soft materials of which gloves are made [19].

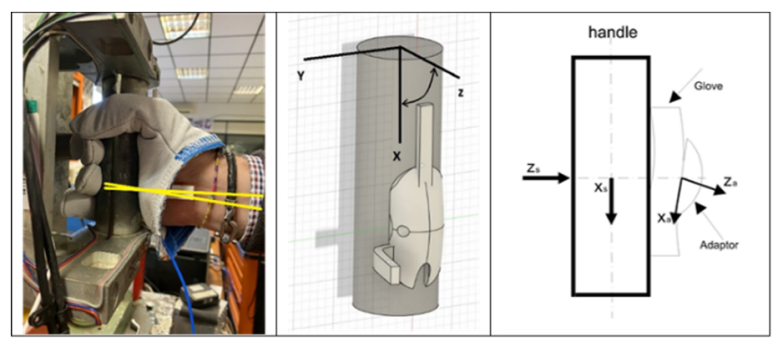

Figure 5: Adaptor's longitudinal and transversal landmarks.

Three healthy male subjects having hand size between 7 and 10, as specified by the EN 420-2003 [24], were recruited for the tests. The shaker was set to produce a vibration of $24 \mathrm{~m} / \mathrm{s}^{2}$ along the Zs axis at $200 \mathrm{~Hz}$. The subjects were asked to maintain the same body and 
hand-arm postures as those required in the standardized glove test [7]. Each subject was asked to maintain a grip force of $80 \pm 5 \mathrm{~N}$ during the tests, without applying any feed force.

The decision to not apply the feed force was taken for minimizing the non-axial vibration produced when the instrumental handle is coupled with the human hand together with a force plate [19]. A visual display of the force signals helped the subjects to maintain the same grip during the tests. An ordinary working leather glove was used for the tests, since its transmissibility is generally close to the unit. Moreover, this glove is not expected to attenuate low-middle frequency vibration [25].

In the first series of tests, the subjects were asked to put the adaptor inside the glove, at the palm level, as recommended by the ISO Standard [7]. No cut on the glove was made in this case for helping to visualize the position of the adaptor. A researcher was in charge to assist the subjects during this phase, providing help in positioning the adaptor inside the glove, so to be aligned as much as possible to the axis of the vibration excitation (Zs). All the subjects and the tests were randomized. Ten tests were made for each of the subjects, each time repeating the entire procedure.

The same protocol was then repeated other two times in order to test the efficacy of both the external referments connected to the adaptors. A little slit was made along the seam of the glove for allowing the longitudinal protrusion to come off. For the other external referment, it was not necessary to cut the glove, since it comes out along the wrist. Also in this case, a three-axial accelerometer was used (SEN $040 \mathrm{~F}$ ) to measure the vibration at the palm level inside the glove.

Some preliminary tests were made for checking the transmissibility of the bare hand adaptor to be sure to minimize the non-axial vibration produced when the instrumented handle is coupled with the human hand. The misalignment of the adaptor, when positioned inside the glove, was evaluated confronting the values of the vibration along $\mathrm{Xa}$ and $\mathrm{Ya}$ axes, both in the case of the use and in the one of no use of the external adaptor protrusions.

\section{RESULTS}

\subsection{Adaptor's transmissibility}

Table 1 reports the average linear and $\mathrm{W}_{\mathrm{h}}$ weighted transmissibility among the considered frequencies for all the tested adaptors, calculated using eqns (2) and (3). Every tested adaptor shows a good response both in linear and in weighted acceleration. The bigger error in linear acceleration was found on the metal adaptor $(+5.2 \%)$, while the one made in polypropylene performed the best result $(+0.3 \%)$. For the printed adaptors the errors were between $+1 \%$ for the Nylon- $50 \%$ and $+2.2 \%$ for the Nylon- $100 \%$.

Table 1: Average linear and $\mathrm{W}_{\mathrm{h}}$ weighted transmissibility of the tested adaptors.

\begin{tabular}{|c|c|c|c|c|c|c|c|c|}
\hline & PLA & PLA & ABS & ABS & NYL & NYL & \multirow{2}{*}{ POLY } & \multirow{2}{*}{ MET } \\
& $100 \%$ & $50 \%$ & $100 \%$ & $50 \%$ & $100 \%$ & $50 \%$ & & \\
\hline $\mathrm{T}_{\text {RL-A }}$ & 1.014 & 1.019 & 1.018 & 1.021 & 1.022 & 1.010 & 1.003 & 1.052 \\
\hline $\mathrm{T}_{\text {RWh-A }}$ & 0.997 & 1.005 & 1.004 & 1.006 & 1.002 & 0.999 & 0.963 & 1.033 \\
\hline
\end{tabular}

Regarding the weighted acceleration, the better transmissibility was performed by the printed adaptors. In this case, the range of error was between $-0.1 \%$ for the Nylon- $50 \%$ and $+0.6 \%$ for the ABS- $50 \%$. The polypropylene adaptor showed the worst behavior producing 
an underestimation of the total transmissibility of $-3.7 \%$, while the metal adaptor performed an overestimation of $+3.3 \%$.

Among the printed adaptors, statistical analysis (ANOVA) reveals no differences at different infill densities for the PLA $(\mathrm{p}=0.06)$ and ABS $(\mathrm{p}=0.65)$, while differences were found among Nylon at $50 \%$ and $100 \%$ of infill density $(\mathrm{p}<0.015)$. Anyway, as reported in Table 1, the differences are more formal than substantial, especially when the acceleration is $\mathrm{W}_{\mathrm{h}}$ weighted.

Regarding the materials, ANOVA analysis confirmed the differences between PLA and Nylon ( $p=0.0029)$ as well as between ABS and Nylon $(p=0.0024)$ but only at $50 \%$ of infill, while no difference was found between PLA-50\% and ABS-50\% $(\mathrm{p}=0.75)$. No differences were found at $100 \%$ infill $(\mathrm{p}=0.15)$ among all the printed materials.

Fig. 6 reports the average transmissibility values $\left(\mathrm{TR}_{(\mathrm{f})-\mathrm{A}}\right)$ of all the tested adaptors at the single frequencies between 10 and $400 \mathrm{~Hz}$. The printed adaptors showed a good response in terms of transmissibility, resulting between 0.95 and 1.05, as prescribed by the Standard ISO 10819:2013, in quite all the analyzed frequency range, as well as the adaptor made in polypropylene. The only cases in which the average transmissibility resulted over $5 \%$ were for $\mathrm{ABS}-50 \%$ at $400 \mathrm{~Hz}\left(\mathrm{TR}_{(\mathrm{f}) \mathrm{A}}=1.06\right)$ and for Nylon- $100 \%$ at $400 \mathrm{~Hz}\left(\mathrm{TR}_{(\mathrm{f}) \mathrm{A}}=1.07\right)$. The metal adaptor on the other hand, revealed an increase of transmissibility over $5 \%$ for most of the frequencies over $80 \mathrm{~Hz}$, with two peaks at 100 and $400 \mathrm{~Hz}$ (TR(f)-A=1.08).

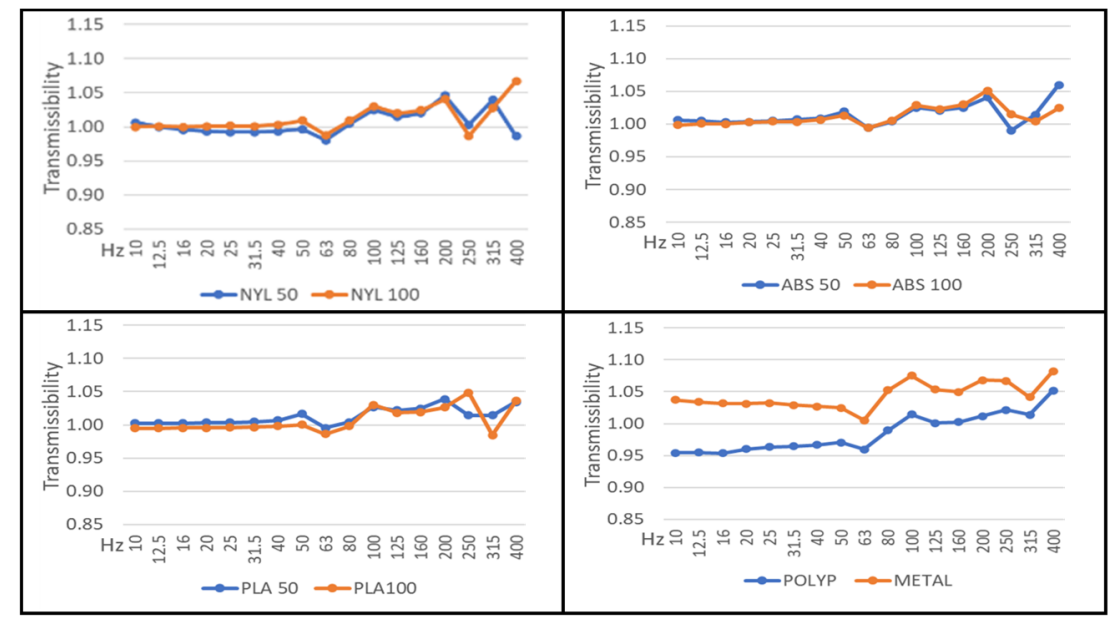

Figure 6: Transmissibility of the adaptors for all the tested frequencies.

All the printed adaptors have quite linear transmissibility, very close to the unit, from 10 to $80 \mathrm{~Hz}$, then the values tend to be nonlinear, with a general tendency to increase as frequencies increase.

T-test was used for the frequency-stratified analysis. Also in this case, no statistical differences were found among the single frequencies between PLA-50\% and PLA-100\%, except at $315 \mathrm{~Hz}(\mathrm{p}=0.001)$. For ABS-50\% and ABS-100\% the only differences were found at $250 \mathrm{~Hz}$ and $400 \mathrm{~Hz}(\mathrm{p}<0.004)$, while in quite all the frequencies, except at $12.5 \mathrm{~Hz}$, the transmissibility of the NYL-50\% defers from NYL-100\% $(\mathrm{p}<0.002)$, showing, in this case, a little better transmissibility of the NYL-50\% at lower frequencies. 
The polypropylene adaptor tends to reduce the transmissibility in the range $10-63 \mathrm{~Hz}$, as well as the metal adaptor amplifies the transmissibility in the range 10-50 Hz. The previous differences were confirmed by the statistical analysis $(p<0.03)$. In the range of frequencies above $100 \mathrm{~Hz}$, the polypropylene adaptor seems to perform better transmissibility compared to those printed, even though the frequency-stratified statistical analysis does not confirm the differences in all the frequencies. The metal adaptor showed higher transmissibility compared to all the others, in quite all the frequency range $(\mathrm{p}<0.002)$.

\subsection{Adaptor's misalignments}

Fig. 7 reports the component of the acceleration along the $\mathrm{Y}$-axis $\left(\mathrm{a}_{\mathrm{y}}\right)$ when the adaptor is positioned inside an ordinary working leather glove, for both the cases in which the external longitudinal protrusion was or was not used. As discussed above, the landmark connected to the adaptor should help the operator to limit the rotation of the accelerometer on the $\mathrm{Y}-\mathrm{Z}$ plane. Even if some components along the Y-axis are always present when the instrumented handle is coupled with the hand [19], when the adaptor is correctly positioned inside the glove, the triaxial accelerometer should reveal mainly only the acceleration provided by the shaker along the Z-axis. On the contrary, if the adaptor is rotated along the longitudinal axis of the instrumented handle, the component of the acceleration increases proportionally along the Y direction [13]. Theoretically, the ratio of the acceleration in the Y direction $\left(a_{y}\right)$ to that in the $\mathrm{Z}$ direction $\left(a_{z}\right)$ is a tangent function of the yaw angle $(\alpha)[16]$ as reported in eqn (4).

$$
\tan \alpha=\frac{\bar{a}_{y}}{\bar{a}_{z}} .
$$

As it can be seen from the graphs reported in Fig. 7, for each of the operators, the component of the acceleration along the $\mathrm{Y}$-axis was found to be greater when the longitudinal landmark was absent, in quite all the tests. Moreover, the acceleration along the Y-axis showed a greater variability among the tests when compared to that measured when the landmark is used.

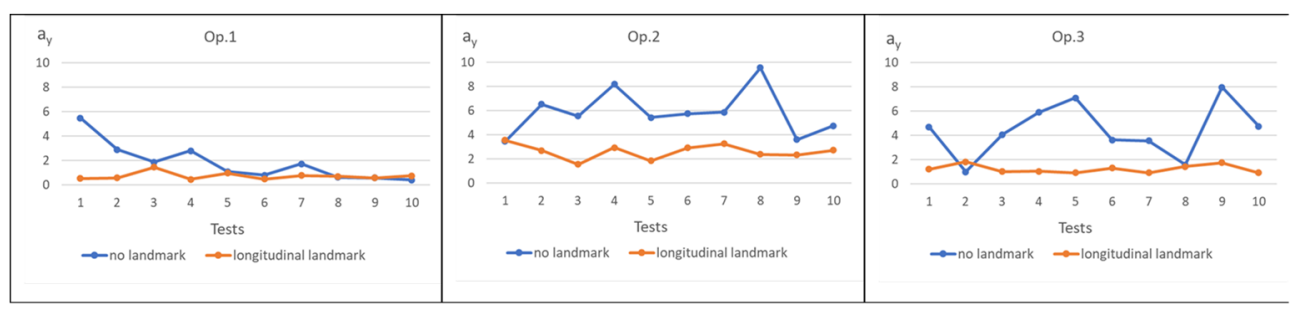

Figure 7: Component of the acceleration along the $\mathrm{Y}$-axis $\left(\mathrm{a}_{\mathrm{y}}\right)$ when the adaptor is positioned inside the glove.

The result supports the idea that the landmark can effectively help the operators for achieving a better alignment of the adaptor inside the glove, limiting the rotation along the $\mathrm{Y}-\mathrm{Z}$ plane.

The ANOVA analysis confirmed the differences among the two series of tests for each of the subjects: operator $1(p=0.043)$; operator $2(p=6.3 E-5)$; operator $3(p=0.0002)$.

Fig. 8 reports the component of the acceleration along the $\mathrm{X}$-axis $\left(\mathrm{a}_{\mathrm{x}}\right)$ when the adaptor was positioned inside the glove for both the situations: the one in which the external 
transversal protrusion was used and the one in which it was or not used. Note that the longitudinal protrusion was present also in these tests since it also helped in avoiding possible rotation of the adaptor on the $\mathrm{X}-\mathrm{Y}$ plane (see Fig. 5).

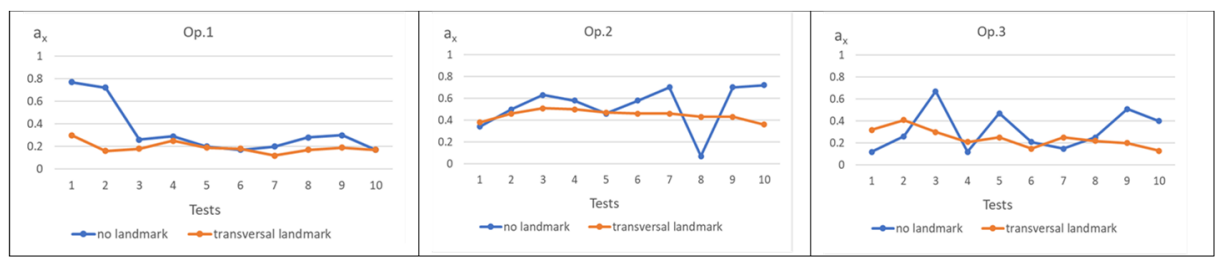

Figure 8: Component of the acceleration along the $\mathrm{X}$-axis $\left(\mathrm{a}_{\mathrm{x}}\right)$ when the adaptor is positioned inside the glove.

As shown in the graphs, the variability of the $a_{x}$ component, among all the tests and for each of the operators, is lower when the transversal landmark is used, compared to the case in which it is not present. That means that the transversal protrusion of the adaptor could help the operator to put the accelerometer in the same position when more tests are performed. Anyway, in this case, the statistical analysis did not confirm the differences among the two series of tests for each of the subjects $(p>0.22)$.

A possible explanation is linked to the thickness of the glove used in the tests. In this case in fact, since the instrumented handle was perfectly straight along the $\mathrm{X}$-axis, supposing that also the adaptor had only a very little rotation on the $\mathrm{X}-\mathrm{Y}$ plane (this was checked looking at the rotation of the longitudinal landmark), possible misalignments can come only from a rotation of the adaptor on the $\mathrm{Y}-\mathrm{Z}$ plane. This rotation can mainly result from the different compression of the material used in the glove for covering the palm of the hand, since the thickness of the glove at the palm level is homogeneous. Anyway, in our case, due to the small thickness of the working leather glove, also the angle between the hand and the forearm, checked looking at the transversal landmark, resulted too little to be easily estimated.

When anti-vibration (AV) gloves are used, since they are thicker and softer, the rotation of the adaptor along the $\mathrm{X}-\mathrm{Z}$ plane could generate greater misalignment between the hand and the forearm, which could be easier checked through the use of the transversal landmark.

\section{CONCLUSIONS}

In the present study, adaptors for measuring hand-arm vibration transmissibility were analyzed when made of different plastic materials (PLA, ABS, and Nylon) at different infill densities (50\% and 100\%). Results show that 3-D printed adaptors, made using fused deposition modeling technique, can be a valid alternative to those on the market. At the middle-low frequencies range $(10 \mathrm{~Hz}-400 \mathrm{~Hz})$ the transmissibility values of the printed adaptors are in line with those of the adaptors found on the market or built using injection molding technique.

No significant differences were found between the transmissibility of those printed at $50 \%$ and $100 \%$ of infill, especially when the acceleration is $\mathrm{W}_{\mathrm{h}}$ weighted. All the printed adaptors have quite linear transmissibility, very close to the unit, from 10 to $80 \mathrm{~Hz}$, then the values tend to be nonlinear, with a general tendency to increase as frequencies increase. The average measured values of the transmissibility were generally inside the range \pm 0.05 in all the tested frequencies. 
Among the analyzed materials, statistical analysis did not show significant differences at $100 \%$ infill, while only the adaptor printed in Nylon showed a little better behavior at frequencies less than $160 \mathrm{~Hz}$ when printed at 50\% infill. Anyway, these differences are very little for having practical repercussions.

Some solutions for better align the adaptor, when used inside gloves, were also evaluated. Starting from the dimensions and the shape reported in the ISO 10819:2013 Standard, a first modification was done, drawing a protrusion along the longitudinal axis of the adaptor. This landmark, coming out from the glove through a little slit made in the seam of the glove between the thumb and index finger, is meant to help for checking possible misalignments of the adaptor, mainly when it rotates on the $\mathrm{Y}-\mathrm{Z}$ plane. Results obtained confronting the component of the acceleration along the y axis $\left(a_{y}\right)$, in both the case of use and not use of the landmark, confirmed its utility. The landmark can also be useful for reducing possible misalignments on the $\mathrm{Y}-\mathrm{Z}$ plane.

A second landmark drawn down along the transversal axis of the adaptor was also tested in order to limit possible misalignments due to a rotation on the $\mathrm{X}-\mathrm{Z}$ plane. These misalignments are generally related to different localized compressions of the glove material at the level of the palm. In this case, even though the results showed a more homogeneous distribution of the acceleration component along the x-axis $\left(a_{x}\right)$, used in this case as an indicator of possible adapter's misalignment, statistical analysis did not confirm the differences between the two series of tests. This can be due to the relatively small thickness of the glove used in the tests that produces little misalignments, too difficult to be checked by the landmark. Some further tests can be carried out using anti-vibration gloves, generally made of thicker and softer materials, to better evaluate the utility of the transversal protrusion applied to the adaptor.

\section{REFERENCES}

[1] Griffin, M.J., Measurement, evaluation, and assessment of occupational exposures to hand-transmitted vibration. Occupational and Environmental Medicine, 54(2), pp. 7389, 1997. https://doi.org/10.1136/oem.54.2.73.

[2] Alfaro Degan, G., Antonucci, A., Coltrinari, G. \& Lippiello, D., Hand-arm vibration induced by the use of radial arm polisher machines. International Multidisciplinary Scientific Geo-Conference Surveying Geology and Mining Ecology Management, SGEM, August(1-2), pp. 127-134, 2020.

[3] Rempel, D., Antonucci, A., Barr, A., Cooper, M.R., Martin, B. \& Neitzel, R.L., Pneumatic rock drills vs. electric hammer drill: Productivity, vibration, dust, and noise when drilling into concrete. Applied Ergonomics, 74, pp. 31-36. 2019. https://doi.org/10.1016/j.apergo.2018.08.005.

[4] ISO 5349-1, Mechanical vibration: Measurements and evaluation of human exposure to hand-transmitted vibration - Part 1: General guidelines. International Standard Organization: Geneva, Switzerland, 2001.

[5] Directive 2002/44/EC of the European Parliament and the Council of 25 June 2002 on the minimum health and safety requirements regarding the exposure of workers to the risks arising from physical agents (vibration).

[6] ANSI S2.70, Guide for the measurement and evaluation of human exposure to vibration transmitted to hand (Revision of ANSI S.3.34-1986), American National Standards Institute (ANSI): New York, 2006.

[7] ISO 10819, Mechanical vibration and shock: Hand arm vibration - Method for the measurement and evaluation of the vibration transmissibility of gloves at the palm of the hand. International Organization for Standardization: Geneva, Switzerland, 2013. 
[8] Dong, R.G., Welcome, D.E., Peterson, D.R., Xu, X.S., McDowell, T.W., Warren, C., Asaki, T. \& Kudernatsch, S., Tool-specific performance of vibration-reducing gloves for attenuating palm-transmitted vibrations in three orthogonal directions. International Journal of Industrial Ergonomics, 44, pp. 827-839, 2014.

http://dx.doi.org/10.1016/j.ergon.2014.09.007.

[9] Budd, D. \& House, R., Examining the usefulness of ISO 10819 antivibration glove certification. Annals of Work Exposure and Health, 61(2), pp. 137-140, 2017. https://doi.org/10.1093/annweh/wxw018.

[10] Song, C.M., Jang, H.-K. \& Chai, J., A comparison of the dynamic properties of transducer adapters for measurement of hand-transmitted vibration. Journal of Mechanical Engineering Science - Part C, 223, pp. 345-351, 2009. https://doi.org/10.1243/09544062JMES1085.

[11] Xu, X.S., Dong, R.G., Welcome, D.E., Warren, C. \& McDowell, T.W., An examination of an adapter method for measuring the vibration transmitted to the human arms. Measurement, 73, pp. 318-334, 2015. http://dx.doi.org/10.1016/j.measurement.2015.05.039.

[12] McDowell, T.W., Dong, R.G., Welcome, D.E., Xu, X.S. \& Warren, C., Vibrationreducing gloves: Transmissibility at the palm of the hand in three orthogonal directions. Ergonomics, 56(12), pp. 1823-1840, 2013. http://dx.doi.org/10.1080/00140139.2013.838642.

[13] Hewitt, S., Assessing the performance of anti-vibration gloves: A possible alternative to ISO 10819-1996. Annals of Occupational Hygiene, 42(4), pp. 245-252, 1998. https://doi.org/10.1016/S0003-4878(98)00030-1.

[14] Xu, X.S., Dong, R.G., Welcome, D.E., Warren, C. \& McDowell, T.W., An examination of the handheld adapter approach for measuring hand-transmitted vibration exposure. Measurement, 47, pp. 64-77, 2014. http://dx.doi.org/10.1016/j.measurement.2013.08.037.

[15] Moschioni, G., Saggin, B. \& Tarabini, M., Uncertainty in hand arm vibration measurements due to the fixation method. 11th International Conference on HandHarm Vibration, Bologna, Italy, 3-7 June, pp. 457-464, 2007.

[16] Xu, X.S., Welcome, D.E., Warren, C.M., McDowell, T.W. \& Dong, R.G., Development of a finger adapter method for testing and evaluating vibration-reducing gloves and materials. Measurement, 137, pp. 362-374, 2019. https://doi.org/10.1016/j.measurement.2019.01.034.

[17] Paddan, G.S., Effect of grip force and arm posture on the transmission of vibration through gloves. Proceedings of the UK Informal Group Meeting on Human Response to Vibrations, MIRA, September, Nuneaton, Warwickshire, UK, 1996.

[18] Smutz, W.P., Dong, R.G., Han, B., Shopper, A.W., Welcome, D.E. \& Kashon, M.L., A method for reducing adaptor misalignment when testing gloves using ISO 10819. Annals of Occupational Hygiene. 46(3), pp. 309-315, 2002. https://doi.org/10.1093/annhyg/mef037.

[19] Dong, R.G., Rakheja, S., Smutz, W.P., Shopper, A.W., Welcome, D. \& Wu, J.Z., Effectiveness of a new method (TEAT) to access vibration transmissibility of gloves. International Journal of Industrial Ergonomics, 30, pp. 33-48, 2002. https://doi.org/10.1016/S0169-8141(02)00076-8.

[20] Dong, R.G., Wu, J.Z., Xu, X.S., Welcome, D.E. \& Krajnak, K., A review of handvibration studies conducted by US NIOSH since 2000. Vibration, 4, pp. 482-528, 2021. https://doi.org/10.3390/vibration4020030. 
[21] Lay, M., Thajudin, N.L.N., Hamid, Z.A.A., Rusli, A., Abdullah, M.K. \& Shuib, R.K., Comparison of physical and mechanical properties of PLA, ABS, and Nylon6, fabricated using fused deposition modeling and injection molding. Composites Part B, 176, pp. 1-8, 2019. https://doi.org/10.1016/j.compositesb.2019.107341.

[22] ISO 5349-2, Mechanical vibration: Measurements and evaluation of human exposure to hand-transmitted vibration - Part 2: Practical guidance for measurement at the workplace. International Standard Organization: Geneva, Switzerland, 2001.

[23] Pitts, P.M., Mason, H., Poole, K.A. \& Young, C.E., Relative performance of frequency weighting $\mathrm{W}_{\mathrm{h}}$ and candidates for alternative frequency weightings when used to predict the occurrence of hand-arm vibration induced injuries. Industrial Health, 50(5), pp. 388-396, 2012. https://doi.org/10.2486/indhealth.MS1381.

[24] EN 420:2003+A1, Protective gloves: General requirements and test methods. The European Standards Body, 2009.

[25] McDowell, T.W., Welcome, D.E., Xu, X.S., Warren, C. \& Dong, R.G., The transmissibility of vibration-reducing gloves at the palm of the hand in three orthogonal directions. Proceedings of the 4th American Conference on Human Vibration, Hartford, Connecticut, USA, June 13-17, 2012. 